PHYSICAL REVIEW D 91, 037501 (2015)

\title{
Effect of $u u$ diquark suppression in proton splitting in Monte Carlo event generators
}

\author{
V. Uzhinsky ${ }^{1,2}$ and A. Galoyan ${ }^{3}$ \\ ${ }^{1}$ CERN, CH-1211 Geneva 23, Switzerland \\ ${ }^{2}$ Laboratory of Information Technologies, JINR, 141980 Dubna, Russia \\ ${ }^{3}$ Veksler and Baldin Laboratory of High Energy Physics, JINR, 141980 Dubna, Russia
}

(Received 27 October 2014; published 3 February 2015)

\begin{abstract}
Monte Carlo event generators assume that protons split into (uu)-diquarks and d-quarks with a probability of $1 / 3$ in strong interactions. It is shown in this paper that using a value of $1 / 6$ for the probability allows one to describe at a semi-quantitative level the NA49 Collaboration data for $p+p \rightarrow$ $p+X$ reactions at $158 \mathrm{GeV} / \mathrm{c}$. The Fritiof (FTF) model of Geant 4 was used to simulate the reactions. The reduced weight of the (uu)-diquarks in protons is expected in the instanton model.
\end{abstract}

DOI: $10.1103 /$ PhysRevD.91.037501

PACS numbers: 24.10.Lx, 13.85.Ni, 14.20.-c

Most of the Monte Carlo event generators of multiparticle production assume that nucleons split into diquarks and quarks in strong interactions. In particular, protons split into (ud)-diquarks and u-quarks with a probability of $2 / 3$, and into (uu)-diquarks and d-quarks with a probability of $1 / 3$. At the same time, there are various physical signatures that the probabilities can be different [1]. For example, it was assumed in the papers [2], as in many other papers, that the (ud)-u configuration completely dominates in the proton wave function. This was motivated be the instanton model [3] of the QCD vacuum. According to that model, quark-quark interactions are flavor-dependent: they are nonzero only if quarks have different flavors. Thus, (uu)-diquarks must be suppressed in protons [4]. The true weight of the (uu)-d configuration can be estimated using the NA49 Collaboration data [5].

The NA49 Collaboration presented high precision data on particle production in $p p$ interactions at $158 \mathrm{GeV} / \mathrm{c}$ including $x_{F}, p_{T}$ and rapidity distributions of various particles $\left(p, n, \pi^{ \pm}, K^{ \pm}, \bar{p}\right)$. As shown in [6,7], Monte Carlo event generators based on the Fritiof model $[8,9]$ cannot satisfactorily describe the data. The most dramatic situation takes place with a description of the proton spectra. A typical prediction for the $x_{F}$-spectrum is shown in Fig. 1 and is presented by the solid thin line.

The Fritiof (FTF) model of Geant4 [10] (release GEANT4.10.0) was used for the simulations. The model considers a diquark as a unit, and assumes the standard splitting of nucleons. As seen, the model overestimates the data at $x_{F} \sim 0.5$, and underestimates the data in the central region $\left(x_{F} \sim 0\right)$. For reproduction of the data at $x_{F} \sim 1$, the high mass diffraction dissociation was simulated with a probability of $10(\mathrm{mb}) / \sigma_{p p}^{\mathrm{in}}$.

The form of the proton spectrum depends, first of all, on the diquark fragmentation function into baryons, $f(z)$. The following fragmentation functions were tested: $1 / z, 1 / \sqrt{z}$, $1, \sqrt{z}, z, z^{2}$. The most acceptable results were obtained with $f(z) \propto \sqrt{z}$. (see dashed red line in Fig. 1).
To improve the prediction in the central region, string inversion was introduced. The Fritiof model assumes that diquarks are leading particles after the splitting. It is not so in the quark-gluon-string model [11,12], where quarks can sometimes be the leading particles. String inversion takes this into account by assuming that a quark is the leading particle in $25 \%$ of cases. This improved the situation in the central region (see dotted blue line in Fig. 1). Now it is clearly seen that the calculations overestimate the multiplicity of the protons.

Changing the weight of the (uu) $-\mathrm{d}$ configuration in protons from $1 / 3$ to $1 / 6$ and using the optimal fragmentation function and the string inversion allow one to reach a semi-quantitative description of the proton spectrum (see solid thick line in Fig. 1). Of course, the description is not perfect but it can be improved in future by a fine tuning of the model parameters.

The proposed approach gives also a possibility to improve the description of neutron and $\Lambda$-hyperon spectra (see Fig. 2).

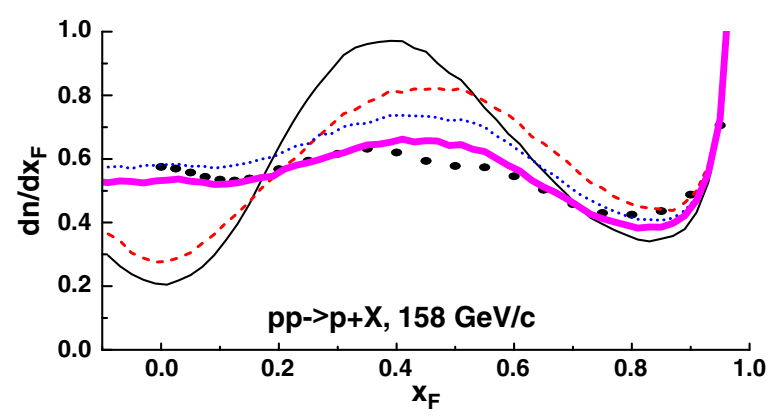

FIG. 1 (color online). $x_{F}$ distributions of protons in $p p$ interactions at $158 \mathrm{GeV} / \mathrm{c}$. Closed points are the NA49 experimental data [5]. Lines are results of FTF model simulations: standard proton splitting (solid black), optimal diquark fragmentation function (dashed red), string inversion (dotted blue), diquark suppression (1/6 instead of $1 / 3)$ including the optimal fragmentation function and the string inversion (solid thick). 

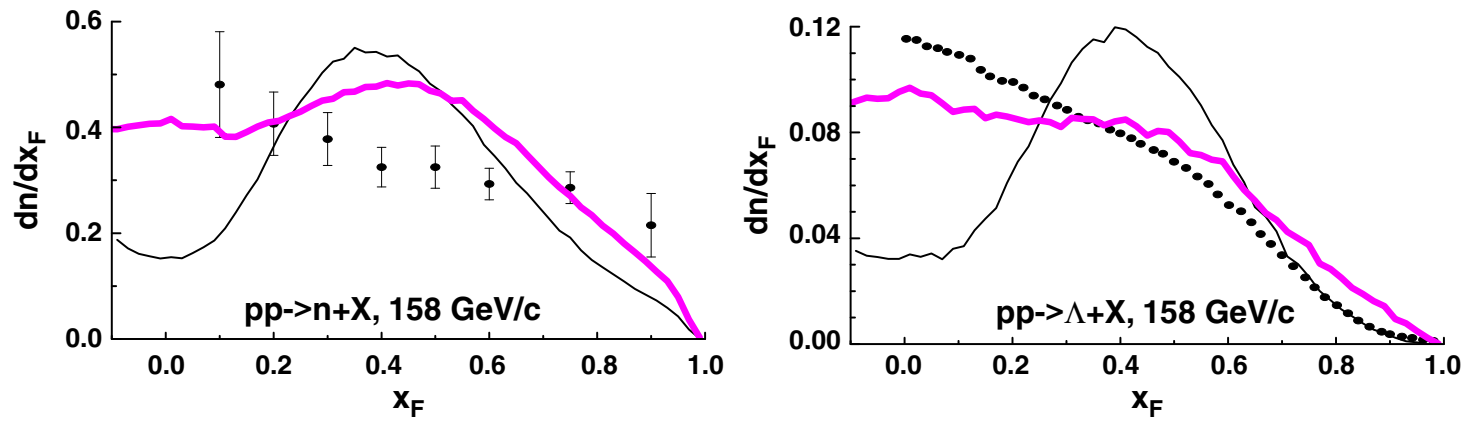

FIG. 2 (color online). $\quad x_{F}$ distributions of neutrons and $\Lambda$-hyperon in $p p$ interactions at $158 \mathrm{GeV} / \mathrm{c}$. Closed points are the NA49 experimental data [5]. Solid thin lines are results of the standard FTF model simulations. Thick lines are results obtained by suppression of the (uu)-diquarks.

In summary, we conclude that the weights of the (uu) $-\mathrm{d}$ configuration in protons and the (dd)-u configuration in neutrons have to be re-considered in Monte Carlo event generators.

\section{ACKNOWLEDGMENTS}

The authors are thankful to N. Kochelev for a useful consideration of the subject of the paper, and J. Harvey, D. H. Wright, A. Ribon for interest to the work and help.
[1] M. Anselmino, E. Predazzi, S. Ekelin, S. Fredriksson, and D. Lichtenberg, Rev. Mod. Phys. 65, 1199 (1993).

[2] N. I. Kochelev, V. Vento, and A. V. Vinnikov, Phys. Lett. B 472, 247 (2000); A. E. Dorokhov and N. I. Kochelev, Z. Phys. C 46, 281 (1990); N. I. Kochelev, Fiz. Elem. Chastits At. Yadra 36, 1157 (2005) [Phys. Part. Nucl. 36, 608 (2005)].

[3] G. 't Hooft, Phys. Rev. D 14, 3432 (1976); D. I. Dyakonov and V. Yu. Petrov, Nucl. Phys. B272, 457 (1986); B245, 259 (1984); Zh. Exp. Theor. Fiz. 89, 751 (1985) [Sov. Phys. JETP 62, 431 (1985)]; T. Schafer and E. V. Shuryak, Rev. Mod. Phys. 70, 323 (1998).

[4] T. Schafer, E. V. Shuryak, and J. J. M. Verbaarschot, Nucl. Phys. B412, 143 (1994).

[5] T. Anticic et al. (NA49 Collaboration), Eur. Phys. J. C 65, 9 (2010); C. Alt et al. (NA49 Collaboration), Eur. Phys. J. C
45, 343 (2006); T. Anticic et al. (NA49 Collaboration), Eur. Phys. J. C 68, 1 (2010).

[6] V. Uzhinsky, arXiv:1308.0736.

[7] V. Uzhinsky, arXiv:1404.2026.

[8] B. Andersson, G. Gustafson, and B. Nilsson-Almqvist, Nucl. Phys. B281, 289 (1987).

[9] B. Nilsson-Almquist and E. Stenlund, Comput. Phys. Commun. 43, 387 (1987).

[10] http://geant4.web.cern.ch/geant4/UserDocumentation/ UsersGuides/PhysicsReferenceManual/fo/PhysicsReference Manual.pdf.

[11] A. B. Kaidalov, Phys. Lett. 116B, 459 (1982); A. B. Kaidalov and K. A. Ter-Martirosyan, Phys. Lett. 117B, 247 (1982).

[12] A. Capella, U. Sukhatme, C. I. Tan, and J. Tran Thanh Van, Phys. Rep. 236, 225 (1994). 